\title{
Correction to: Polythiophene-Chitosan Magnetic Nanocomposite as a Novel Sorbent for Disperse Magnetic Solid Phase Extraction of Triazine Herbicides in Aquatic Media
}

\author{
Shokooh Ehteshami ${ }^{1}\left[\right.$ ] Alireza Feizbakhsh ${ }^{1}$
}

Published online: 7 August 2018

๑) Springer-Verlag GmbH Germany, part of Springer Nature 2018

\section{Correction to: Chromatographia (2016) 79:1177-1185}

https://doi.org/10.1007/s10337-016-3134-9

The authors would like to call the reader's attention to the fact that unfortunately the order of authors' names was wrong in the original publication.

The corrected order is given below.

Shokooh Ehteshami and Alireza Feizbakhsh

Alireza Feizbakhsh

Ali.Feizbakhsh@iauctb.ac.ir; Ali.fb1973@yahoo.com

Shokooh Ehteshami

Sho.ehteshami.sci@iauctb.ac.ir

1 Analytical Chemistry Laboratories, Department of Chemistry, Islamic Azad University, Central Tehran branch, Tehran, Iran 